\title{
A model for mutation in bacterial populations
}

\author{
R. Donangelo ${ }^{1}$ and H. Fort ${ }^{2}$ \\ ${ }^{1}$ Instituto de Física, Universidade Federal do Rio de Janeiro, C.P. 68528, 21945-970 Rio de Janeiro, Brazil. \\ E-mail:donangel@if.ufrj.br \\ ${ }^{2}$ Instituto de Física, Facultad de Ciencias, Universidad de la Repblica, Iguá 4225, 11400 Montevideo, Uruguay. E-mail: \\ hugo@fisica.edu.uy
}

\begin{abstract}
We describe the evolution of E.coli populations through a Bak-Sneppen type model which incorporates random mutations. We show that, for a value of the mutation level which coincides with the one estimated from experiments, this model reproduces the measures of mean fitness relative to that of a common ancestor, performed for over 10,000 bacterial generations.
\end{abstract}

\section{PACS numbers: 05.65.+b, 87.23.-n, 89.75.Fb}

The last decade has seen a renewed interest in the study of biological evolution. Besides the painstaking work of analyzing fossil records, spanning $10^{8-9}$ years, there are now experiments performed by Lenski and coworkers with E.coli, which already comprise tens of thousands of generations [1] . This has opened an entire "experimental evolution" area, and their data are extremely useful to study the long-term evolutionary dynamics of populations.

We first briefly review the essentials of Lenski's experiment. It considered 12 initially identical populations, each of them founded by a single cell from an asexual clone, propagating in similar environments during 1500 days, in the following manner. At the beginning of each 24 hour period, an initial batch of around $5 \times 10^{6}$ bacteria is placed in a growth medium, and, 24 hours later, when the population has increased by a factor of about 100 , which implies $\log _{2} 100 \approx 6.6$ generations, the process is repeated by starting a new batch with $1 \%$ of the bacteria present.

The mean cell volume and mean fitness of bacterial populations relative to the ancestor $\langle R F\rangle$ were measured every 100 generations. The $\langle R F\rangle$ of these populations, in its $g$-th generation is measured by placing a sample of each of them in contact with its original ancestor (unfreezing a sample taken at time 0 , the first generation), and measuring the ratio of their rates of increase. In all the experiments the $\langle R F\rangle$ shows a rapid increase for $\approx 2000$ generations after its introduction into the experimental environment, and then becomes practically constant. The average asymptotic value of the relative fitness is $\langle\overline{R F}\rangle_{\infty} \simeq 1.48$ 迎 (the bar denotes the average over the 12 populations). This behavior may be parameterized by an hyperbolic fit, $f=(A+B g) /(C+D g)$, where $f$ represents the relative fitness of the $g$-th generation and $A, B, C, D$ are constants.

On the theoretical side, several approaches to the evolutions of species in interactive systems have been pro- posed: the well known models of Kauffman and collaborators for co-evolving species operating at the edge of criticality [2], models inspired on them [3], [4], and the Bak-Sneppen (BS) model [5], among others.

An essential ingredient of evolution theory, complementing the natural selection mechanism, is the existence of spontaneous mutations which produce hereditary differences among organisms. Such an ingredient is not explicitly considered in the standard BS model, but it is clearly an essential mechanism in the evolution of bacterial cultures.

In this work we modify the BS model in order to include random mutations so as to explain the E.coli results. The reasons for constructing such a model are twofold. First, it was experimentally found that the 12 E.coli replicate populations approached distinct fitness peaks (different asymptotic $\langle R F\rangle$ into a band from $\approx 1.4$ to $\approx 1.6)[7]$, supporting the multi-peaked fitness landscape of the kind assumed in BS-type models. Second, as the initial populations were identical, and the environment for bacterial growth was kept constant, the evolution of these quantities resulted solely from spontaneous random genetic changes and competition between the different cell varieties resulting from those changes.

The model thus assumes two kinds of changes, one arising from the disappearance of the less fit strains and another, completely random, that may be attributed it e.g. to errors in the replication mechanism. All these changes are associated, in the model, to real mutations in the genome. In the case of the fitness driven changes, such mutations appear as a two-step process: the extinction of a bacterial strain, and its substitution by another, as in the original BS model. The new random mutations are associated to changes in the genoma unrelated to any selection process.

Some clarifying remarks concerning the proper interpretation of the model in the context of E.coli experiment are necessary:

i) Despite the fact that the original BS model considered evolution in a coarse grained sense (an entire species was represented by a single fitness parameter), here we will describe a system of evolving bacterial populations rather than whole species.

ii) The focus of the BS model was to study the dynamic equilibrium properties of an ecology, i.e. its SelfOrganized Critical (SOC) behavior after the initial transient. The data of [1] consider explicitly the transient evolution starting from the first bacterial generations. In 
particular, changes are observed to be larger for the first 2000 generations, and then gradually taper off. Therefore, we consider the evolution of the system from its very initial state, and not after it has equilibrated.

iii) In our model we consider a number of cellular automata $N$, which, for practical purposes, must take values much smaller than the number of E.coli in Lenski's experiment $\left(5 \times 10^{6}-5 \times 10^{8}\right)$. Below we show that the model has scale invariance properties that justify this assumption.

As in the case of BS, the model consists of a onedimensional cellular automata of size $N$, with cells labelled by a subindex $i=1, \ldots, N$. Therefore each of these $N$ cells represents a group of bacteria, and not single individuals. In other words, the system in the model is a coarse-grained representation of the cell population. Each vector cell is characterized by a real number between 0 and $1, B_{i}$. This parameter may be interpreted as measuring the fitness of the "species", i.e. a barrier against changes.

In order to emulate the experimental condition that each of the 12 populations was initiated by a single cell from an asexual clone, we start with the same barrier for all cells, $B_{i}=0.5$. The dynamics of the model consists in performing the some operations at steps corresponding to the time needed for an average E.coli to divide, $i$. $e$. a generation. Those operations are the following: at each step, corresponding to the doubling time of the bacteria: a) eliminate the cell with the lowest fitness b) eliminate, on the average, $Q$ other cells c) replace the barriers associated to the cells eliminated by random numbers generated with a uniform distribution in the interval [0,1], as in the BS model

Results do not depend on the choice of distribution employed in operation c). Operations a) and b) are associated to either fitness driven or random mutations, respectively.

We should remark that in order to simulate the experiment, one would need to double the total number of cells at the end of each step, assigning copies of the barriers associated to the existing cells to the ones created. Furthermore, every $G \approx 6-7$ steps, the population should be reduced to its original value $N$ by selecting at random this number of cells among the total population. However, since the mutation probability is found to be constant and independent of the size of the population, scaling properties [11 allow us to avoid these population doublings and reductions, keeping the number of cells constant. Because of this simplification, the model becomes formally equivalent to the mean field version of the BS model (MFBS) [6] However, here the $Q$ barrier changes in c) are interpreted as random mutations, and not as changes to neighbors of the least fit specimen in the population as in the MFBS model.

Since, as in the BS model, after a transient, the model fitness barrier distribution self-organizes in a step at $B_{c}=1 /(1+Q)$, its asymptotic mean fitness is
$\left(B_{c}+1\right) / 2=(Q+2) /(Q+1)$, while the mean fitness of the original uniform barrier distribution is 0.5. Therefore, in this model the asymptotic relative fitness to the ancestor is

$$
\langle R F\rangle_{\infty}=\frac{Q+2}{Q+1} .
$$

We select the value of $Q$ so as to adjust $\langle\overline{R F}\rangle_{\infty} \simeq$ 1.48. Hence, from (1) we obtain $Q \simeq 1$.1. It is interesting to note that the model suggests an approximately equal number of fitness driven and random mutations for the E.coli under the conditions of Lenski's experiment.

While $Q$ determines the asymptotic value of $\langle R F\rangle_{\infty}$, the number of cellular automata, $N$, is determined by the empirically observed mutation rate per replication $\mu$, which was estimated as $\mu \approx 0.002$ per replication [8], [9]. Since in the model we have on the average $Q+1=2.1$ changes per generation, then the number of cells in the simulation should be $N \approx 1000$. In this way, the two parameters of the model, $Q$ and $N$, are fixed so as to reproduce the experimentally observed asymptotic fitness and mutation rate found in the experiment. The simulations were performed for $Q=1.1$ and for several values of $N$ in the interval $500 \leq N \leq 2000$.

The agreement between the model and experiment is quite reasonable. In Fig. 1 we plot the $\langle R F\rangle$ trajectory every 500 generations for $N=1000(\square)$ and $N=1500$ $(\triangle)$ and 3 of the Lenski et al. best hyperbolic fits to 3 (of the 12) sets of data for the E.coli experiments.

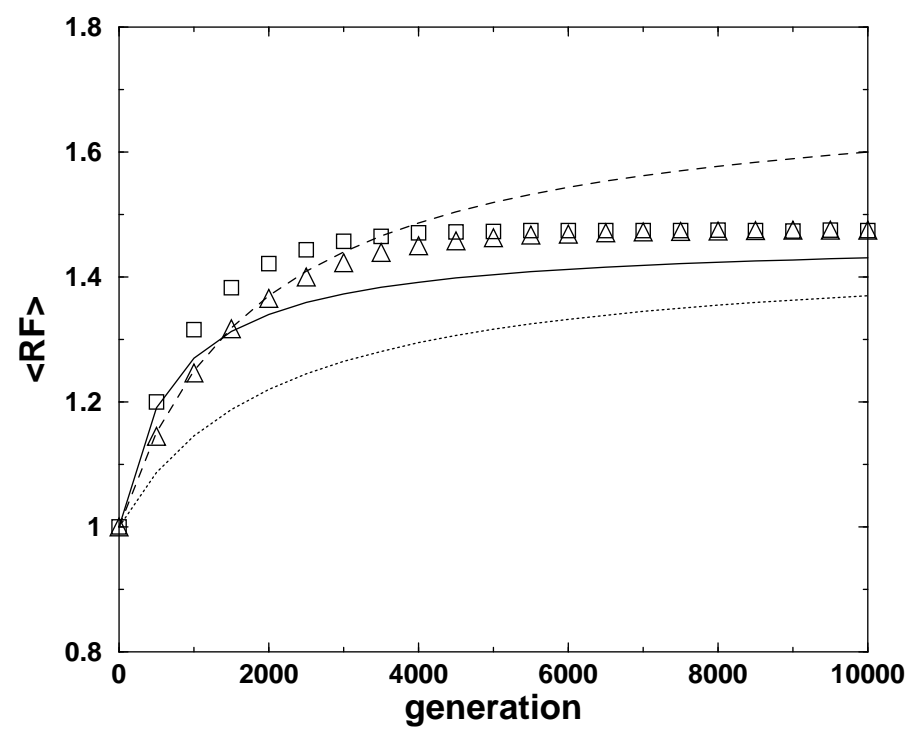

FIG. 1. Trajectories for mean fitness relative to the original ancestor during 10,000 generations. Averages from 1000 numerical simulations for $Q=1.1$ for $N=1000$ (squares) and $N=1500$ (triangles) compared with the 3 best hyperbolic fits (lines) to data of 3 of the 12 corresponding experiments. 
In order to analyze the initial rapid grow of $\langle R F\rangle$, in Fig. 2 we plot the experimental data and the model results for the first 2000 generations, every 100 generations. Once again notice the overall agreement of the model with experimental E.coli data.

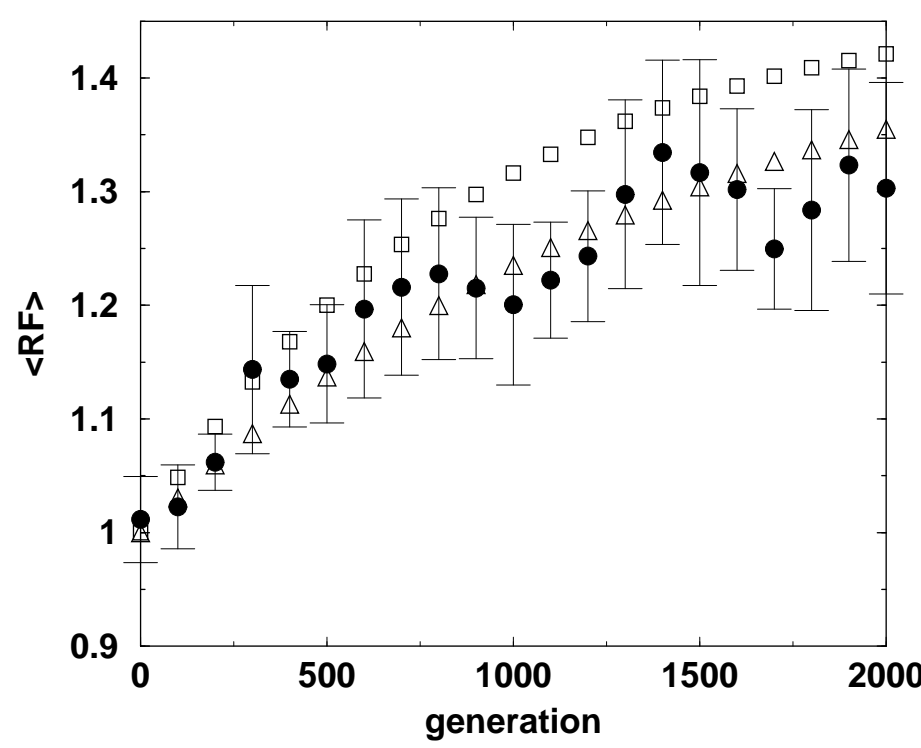

FIG. 2. Finer scale analysis of the trajectories of the mean fitness relative to the ancestor for the 2000 initial generations. Experimental data (filled circles), and model averages from 1000 numerical simulations with $Q=1.1: N=1000$ (squares) $N=1500$ (triangles). The standard deviations of the experimentally measured relative fitness are indicated as error bars in the data points. The model calculations have negligible dispersion in the scale of the plots presented in this work.

It was suggested in [1] that periods of stasis, characteristic of punctuated evolution, might be present in the data. Although the relatively large experimental error bars could make the data consistent with a monotonic increase like the one predicted by the model, we believe that further studies are needed to settle this issue.

The results presented thus far assume that there is no neighbor relation between different strains of E.coli in the system. However, as in other ecological system interdependencies among species arise, one should explore the possibility that also in this case they exist. To do this, we think it is illustrative to present here also the results of a variation of the standard BS-type model. In this variation, as in the original model, at each time step the changes occur at the cell with lowest barrier and its two neighbors. In addition to this fitness driven form of evolution we include, with probability $p$ per time step, a similar change in a randomly chosen cell. In this way the number of changes per time step is $Q=2+p$ (the two neighbors of the cell with minimum barrier plus, with probability $p$, a cell at a random location). We denote this version of the model as $\mathrm{BS}+p$.
The stationary properties of this $\mathrm{BS}+p$ model will be presented elsewhere [10]. We have observed that the barrier distribution self-organizes into a step function at a position $B_{c}$ which decreases as the parameter $p$ increases from $B_{c} \simeq 0.667$ for $p=0$ (the standard BS model) to $B_{c} \simeq 0.22$ for $p=1$. Thus $\langle R F\rangle_{\infty}$ lies in the interval between 1.22 (for $p=1$ ) and 1.667 (for $p=0$ ). We found that $\langle\overline{R F}\rangle_{\infty} \simeq 1.48$ may be adjusted taking $p \simeq$ 0.2 . Hence, here the purely random mutations are taken with a weight proportional to 0.2 , while those mutations related to natural selection are proportional to 3 .

In this $\mathrm{BS}+p$ version, in an analogous way as it happens in the MFBS version, while $p$ determines the asymptotic value of $\langle R F\rangle_{\infty}$, the number of cellular automata, $N$, is determined to fit with the estimated $\mu \approx 0.002$. Since, we now have, on the average, 3.2 changes per time step, in order to get roughly the same mutation rates in the simulation and in the experiment we should take $N \simeq 1500$. In Fig. 3 we plot the $\langle R F\rangle$ trajectory every 500 generations for $N=1000(\square)$ and $N=1500(\triangle)$ and 3 different hyperbolic fits of [1]. Notice the good agreement with the Lenski et al. hyperbolic fit to data corresponding to the "A-1" experiment [7].

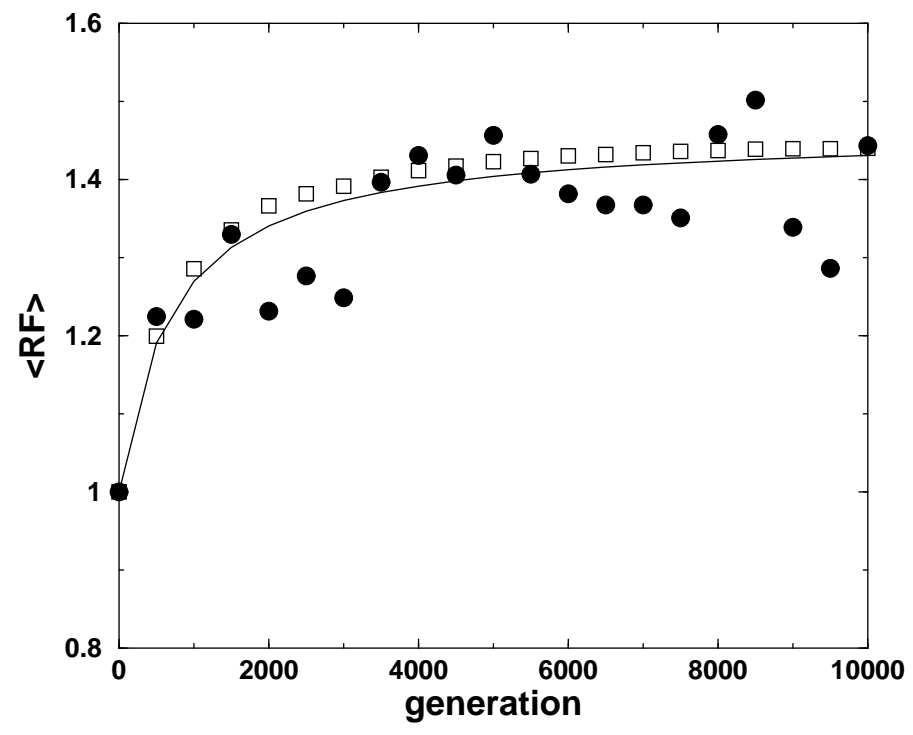

FIG. 3. Trajectories for mean fitness relative to the original ancestor during 10,000 generations, in the $\mathrm{BS}+p$ model. Averages from 1000 numerical simulations for $p=0.2$ for $N=1500$ (squares) compared with the data (filled circles) and their best hyperbolic fit (line). See text for further details.

In the case of the E.coli, the MFBS model appears, in principle, more reasonable, as it does not seem plausible to have interdependencies among closely related bacterial strains. The $\mathrm{BS}+p$ model could be applicable to other situations, where different microorganisms coexist and are interdependent. Our point here was to show that 
it is not possible to distinguish between the two models based solely on the measurements of the evolution of the fitness.

To conclude, when considered during the transient from the initial ordered distribution, BS models with random mutations were shown to qualitatively reproduce the experimental results of Lenski and co-workers. The inclusion of random mutations, besides making more realistic the models, is required to get quantitative agreement with the experimental results, both for the transient and the asymptotic regime. While both fitness driven and random mutations were shown to be needed, their relative importance remains an open question. One should remark that the calculations presented here are just an starting point in the exploration of this complex biological system. In particular, the existence of stasis regions, suggested by the data, remains another open issue.

Work supported in part by PEDECIBA (Uruguay)

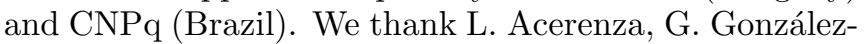
Sprinberg and K. Sneppen for valuable discussions and suggestions.

[1] R. Lenski and M. Travisano, Proc. Natl. Acad. Sci. 91, 6808 (1994).

[2] S. A. Kauffman and S. J. Johnsen, J. Theor. Biol. 149, 467 (1991).

[3] H. Flyvberg and B. Lautrup, Phys. Rev. A 46, 6714 (1992).

[4] P. Bak, H. Flyvbjerg and B. Lautrup, Phys. Rev. A 46, 6724 (1992).

[5] P. Bak and K. Sneppen, Phys. Rev. Lett. 71, 4083 (1993); K. Sneppen, P. Bak, H. Flyvbjerg and M. H. Jensen, Proc. Natl. Acad. Sci. 92, 5209 (1995).

[6] H. Flyvbjerg, P. Bak and K. Sneppen, Phys. Rev. Lett. 71, 4087 (1993);

[7] A summary data from the long-term evolution experiment of Lenski and co-workers can be found at http://myxo.css.msu.edu/ecoli/summdata.htm]

[8] J. W. Drake, Proc. Natl. Acad. Sci. 88, 7160 (1991).

[9] S. F. Elena, V. S. Cooper and R. Lenski, Science 272, 1802 (1996).

[10] R. Donangelo and H. Fort, to be published.

[11] Henrik Jeldtoft Jensen. Self-Organized Criticality: emergent complex behavior in physical and biological systems, Cambridge University Press, 1998, eq. 5.39. 